\title{
Evaluación de la rentabilidad del manejo en bosques secundarios de canelo (Drimys winteri) en la Cordillera de la Costa de Valdivia, Chile
}

\author{
Financial evaluation of management in secondary forests of canelo \\ (Drimys winteri) in the Coastal Range of Valdivia, Chile \\ Celso Navarro Cárcamo ${ }^{a *}$, Miguel Angel Herrera ${ }^{\mathrm{b}}$, Fernando Drake Aranda ${ }^{\mathrm{c}}$, Pablo J Donoso ${ }^{\mathrm{d}}$ \\ *Autor de correspondencia: aUniversidad Católica de Temuco, Escuela de Ciencias Forestales, \\ Rudecindo Ortega 02950, Temuco, Chile, tel.: 56- 45- 205545, cnavarro@uct.cl \\ bUniversidad de Córdoba, Córdoba, España. \\ 'Universidad de Concepción, Facultad de Ciencias Forestales, Concepción, Chile. \\ dUniversidad Austral de Chile, Facultad de Ciencias Forestales y Recursos Naturales, Valdivia, Chile.
}

\begin{abstract}
SUMMARY
Young secondary forests of Drimys winteri have excellent growth and regeneration, and cover an area of 230,000 hectares in Chile. However, there is no supply of high-value timber to feed the growing market opportunities. The objective of this study was to evaluate the profitability of thinning trials in secondary forests of Drymis winteri in a medium-productivity site. The thinning treatments were considered late thinning, and were named as moderate thinning (E1), heavy (E2) and heavy (E3), in addition to a control treatment (E4). The yield was determined using the net present value (NPV), land expected value (LEV), and modified internal rate of return (MIRR), incorporating discount rates of $6 \%, 8 \%$ and $10 \%$ and low administration costs in two scenarios, no costs before thinning (S1) and costs since the establishment of the stand (S2). The results for the NPV and VPS in S2 were negative, in contrast with S1, which doubled its profitability compared with the control treatment. The E2 treatment had the highest profitability in the S1 case, with a NPV of USD 2,724 $\mathrm{ha}^{-1}$, a VPS of USD 3,771 for a $6 \%$ rate, and a MIRR of $19 \%$. For an operational level the moderate thinning treatment (E1) is desirable since it reduces the risk of windfall, generates the highest yield in volume, and profitability values slightly lower than E2.
\end{abstract}

Key words: forest economy, Drimys winteri, intermediate cut, profitability.

\section{RESUMEN}

Los bosques secundarios de canelo presentan excelentes crecimientos y regeneran fácilmente en forma natural, alcanzando una superficie de 230.000 hectáreas en Chile. A pesar de ello, no existe una oferta de madera que permita aprovechar las oportunidades de los mercados nacionales e internacionales. El objetivo de este estudio fue evaluar la rentabilidad de ensayos de raleo en bosques secundarios de Drimys winteri en un sitio de productividad media. Los tratamientos corresponden a raleos aplicados tardíamente, denominados como raleo moderado (E1), fuerte (E2 y E3) y un tratamiento control (E4). La rentabilidad se determinó a través del valor actual neto (VAN), valor económico del suelo (VES) y la tasa interna de retorno modificada (TIRm) incorporando tasas de descuento de $6 \%, 8 \%$ y $10 \%$; se consideraron costos de administración bajo dos escenarios: sin costo antes de las intervenciones (S1) y con costo desde el establecimiento del rodal (S2). Los resultados para el VAN y VPS en S2 fueron negativos, a diferencia del S1 que duplicó al menos su rentabilidad respecto de no intervenir los bosques secundarios. El tratamiento E2 presentó la mayor rentabilidad para el supuesto S1 con un VAN de US\$2.724 ha ${ }^{-1}$, un VPS de US\$ 3.771 para una tasa de $6 \%$ y una TIRm de $19 \%$. Es recomendable para un nivel operacional el tratamiento de raleo moderado (E1), ya que reduce el riesgo de caída por viento, genera el mayor rendimiento en volumen y presenta cifras de rentabilidad levemente inferiores a E2.

Palabras clave: economía forestal, canelo, cortas intermedias, rentabilidad.

\section{INTRODUCCIÓN}

En Chile existen 4,4 millones de hectáreas de bosques secundarios con gran potencial de intervención a corto plazo, bajo criterios de manejo sustentable (CONAF et al. 1997). Las productividades varían desde $8 \mathrm{~m}^{3} \mathrm{ha}^{-1}$ año $^{-1}$ a $20 \mathrm{~m}^{3}$ ha ${ }^{-1} \mathrm{año}^{-1}$ y no han requerido para su desarrollo de inversión alguna, sino sólo del espacio físico y el tiempo transcurrido entre su establecimiento y la actualidad (Emanuelli
1996). De estas formaciones, los bosques secundarios de Drymis winteri J. R. et G. Forster constituyen una de las asociaciones vegetacionales de segundo crecimiento más abundantes de las regiones de Los Lagos y de Los Ríos de Chile $\left(40^{\circ}\right.$ a $\left.44^{\circ} \mathrm{S}\right)$, alcanzando una superficie de 230.000 ha (CONAF et al. 1997). Estos bosques se forman luego de eventos catastróficos como incendios y talas, que generan condiciones en el terreno bajo las cuales las especies que se establecen con posterioridad no tienen competencia de 
árboles cercanos (Donoso 1999), particularmente en sitios bajos, pobremente drenados y húmedos.

Las tasas de crecimiento en bosques secundarios de D. winteri dependen de la clase de sitio en el cual se encuentren, alcanzando crecimientos entre 10 a $16 \mathrm{~m}^{3}$ $\mathrm{ha}^{-1}$ año $^{-1}$ en los mejores sitios (Calquín 1986). En los sitios más productivos se recomienda realizar tratamientos silviculturales para potenciar su productividad, obtener productos de alta calidad y mejorar la rentabilidad de los bosques (Navarro et al. 1997). En el estado actual de los bosques secundarios, intervenciones silviculturales como raleos adquieren mayor importancia, debido a sus altas densidades, alta mortalidad por competencia, estructuras diamétricas exponenciales negativas y buenas respuestas a intervenciones silviculturales (Navarro et al. 1997, Reyes et al. 2009). Adicionalmente a estas ventajas, estudios de mercado recientes desarrollados por el Instituto Forestal le atribuyen a la madera de $D$. winteri buenas perspectivas, particularmente en Estados Unidos (INFOR 2005). Basado en lo anterior, realizar un buen manejo de estos bosques secundarios podría ser rentable, especialmente si se determinan las mejores opciones silviculturales y adecuadas condiciones en los mercados nacionales e internacionales.

A pesar de las ventajas que presentan estos bosques secundarios, no se conoce la rentabilidad de opciones silviculturales para la producción de trozos de calidad. Para que el manejo de estos bosques sea sustentable, éste debe retornar un valor económico al inversor, que se deriva de una mezcla compleja de costos, ingresos, problemas de calidad y demanda. Estos factores son principalmente determinados a través de influencias fuera del bosque, y es el resultado de las tendencias en la política nacional e internacional y los eventos económicos que forman el ambiente comercial global (Acuña y Drake 2003).

Se plantea como hipótesis que las intervenciones de intensidad moderada aumentan el rendimiento en volumen total y la rentabilidad, maximizando de esta forma la producción económica del sitio. Los objetivos de este estudio son a) evaluar la rentabilidad del manejo silvicultural en bosques de segundo crecimiento de $D$. winteri en Chile, orientado a la producción de trozos para aserrío y debobinado, y b) analizar la tecnología silvicultural factible de aplicar mediante un modelo de programación dinámica que maximiza la tecnología silvicultural en términos de rentabilidad y producción física.

\section{MÉTODOS}

Área de estudio. El área de ensayo se localizó en la Cordillera de la Costa de Valdivia en Chile, en el lugar denominado Hueicolla $\left(40^{\circ} 07^{\prime}\right.$ S y $73^{\circ} 23^{\prime}$ O). Predomina el tipo forestal siempreverde según la tipología forestal determinada por Donoso (1981), con presencia importante en los sectores de menor pendiente del subtipo bosques secundarios de $D$. winteri.
El clima en esta área corresponde al tipo oceánico templado-húmedo (Di Castri y Hajek 1976), caracterizándose por altas precipitaciones y temperaturas moderadas debido a la influencia oceánica. La precipitación media anual cerca del nivel del mar, donde se ubican los bosques secundarios estudiados, alcanza alrededor de $3.500 \mathrm{~mm}$, pudiendo llegar hasta $6.730 \mathrm{~mm}$ en la parte alta de la Cordillera de la Costa (Oyarzún et al. 1998).

Los suelos corresponden a la serie Hueicolla, la cual se encuentra al sur del río Valdivia, entre 100 y $1.000 \mathrm{~m}$ s.n.m. Son suelos con grandes variaciones de espesor, predominando los moderadamente profundos, de origen metamórfico, de texturas que varían entre moderadamente finas en superficie a finas en profundidad. El arraigamiento es bueno hasta los $46 \mathrm{~cm}$, siendo en general suelos muy ácidos. La materia orgánica es abundante en la superficie pero disminuye bruscamente en profundidad. Estos suelos además se destacan por su bajo contenido de fósforo y alto contenido de aluminio (Morales et al. 1988, Donoso y Nyland 2005).

La topografía existente en el área es abrupta con pendientes que varían entre 0 y $80 \%$, predominando las pendientes superiores a $30 \%$. El ensayo se encuentra en la parte baja de la ladera occidental de la Cordillera de la Costa entre los 41 y $158 \mathrm{~m}$ s.n.m. y pendientes entre 15 y $40 \%$ en exposiciones sur, suroeste y oeste.

Antecedentes del ensayo y mediciones. Los bosques secundarios de $D$. winteri estudiados corresponden a bosques puros, donde en condiciones sin manejo $D$. winteri aporta el 81,2\% del número de árboles, $81,4 \%$ del área basal y $84,1 \%$ del volumen total (Navarro et al. 1997). Las especies acompañantes no fueron analizadas individualmente, siendo las más frecuentes Luma apiculata (D. C.) Burret, Amomyrtus luma (Mol.) Legr. et Kausel, Embothrium coccineum J. R. et. G. Forster, Eucryphia cordifolia Cav., Laurelia philippiana Looser. y Ovidia pillo pillo (C. Gray) Hohen. ex CFW Meissn.

Los parámetros dasométricos promedios alcanzan cifras de 6.174 árboles por hectárea, $57,7 \mathrm{~m}^{2} \mathrm{ha}^{-1}$ en área basal y $354,7 \mathrm{~m}^{3} \mathrm{ha}^{-1}$ en volumen. La edad promedio del bosque al momento de establecerse el ensayo era de 30 años el año 1985 (Navarro et al. 1999).

Tecnologías silviculturales. Parte importante de los bosques secundarios de $D$. winteri en Chile se encuentran en estados de desarrollo biológicamente tardíos para realizar un primer raleo, ya que el máximo crecimiento anual periódico se alcanza entre los 8 a 14 años en los sitios de productividad media (Navarro 1993), siendo importante discutir las tecnologías silviculturales factibles de aplicar en estos bosques con edades promedio de 30 años.

El estudio se basó en un ensayo de intensidad de raleo instalado en 1985 que es el de mayor data documentado en Chile, establecido en un sitio de productividad media, con crecimientos entre 6 y $10 \mathrm{~m}^{3} \mathrm{ha}^{-1} \mathrm{año}^{-1}$ y altura dominante 
de $16 \mathrm{~m}$ a los 35 años de edad. Los tratamientos aplicados según Nyland (2002) corresponden a raleos por lo bajo tipo $\mathrm{C}$ y D, denominados para este estudio como raleo moderado (E1), fuerte (E2 y E3), más un tratamiento testigo sin raleo (E4). Estos corresponden respectivamente a espaciamientos promedios entre árboles de dos, tres y cuatro metros. Se realizaron tres repeticiones por tratamiento, con un total de 12 parcelas de $600 \mathrm{~m}^{2}$ cada una. El año 1996, luego de 10 años del primer raleo, el tratamiento moderado fue nuevamente intervenido, dejando una densidad equivalente al raleo fuerte (E2), es decir, a un espaciamiento promedio de $3 \mathrm{~m}$ entre árboles.

En todos los tratamientos se privilegió dejar los individuos pertenecientes al estrato dominante y de excelente calidad de la especie $D$. winteri. Al respecto, Navarro et al. (1999) proponen para estos sitios, que poseen crecimientos medios anuales de $0,45 \mathrm{~cm}$ en diámetro y $10,1 \mathrm{~m}^{3}$ en volumen, realizar las primeras intervenciones mucho antes, cuando el rodal alcanza el máximo crecimiento anual periódico de $0,92 \mathrm{~cm}$ año ${ }^{-1}$, lo que se produce entre los 10 y 12 años de edad. Por lo tanto, este estudio evalúa diferentes intensidades de raleo tardíos en bosques secundarios de $D$. winteri y analiza la tecnología silvicultural factible de implementar, incorporando el tipo de corta final o de regeneración posible de aplicar legalmente. En el caso del tipo forestal siempreverde, al que pertenecen estos bosques secundarios, existen legalmente en Chile dos métodos de corta, cortas de protección y cortas de selección (Donoso 1981). Se consideró para este estudio como método de corta final el de protección en fajas alternas recomendado por Donoso (1999) como alternativa de corta de regeneración para los bosques del tipo forestal siempreverde, luego de un par de décadas de evaluación de ensayos experimentales. No obstante, el método de corta final para efectos de comparar los tratamientos de raleo que se discuten en este documento no tiene efecto, ya que se toma como una constante para la tecnología silvícola; aunque sí afecta la magnitud del resultado en términos financieros y físicos.

La dinámica natural de estos bosques secundarios dará origen a bosques adultos mixtos siempreverdes donde $D$. winteri participa como una especie secundaria en términos comerciales (Donoso 1981), ello debido a su baja participación en el número de árboles. Al respecto, Navarro et al. (1997) señalan que proyectar bosques donde predomine $D$. winteri en sitios de productividad media implica cosechar los árboles a edades no mayores a 80 años a causa de la pérdida de calidad de la especie.

Mediciones y procesamiento de datos. Las mediciones se realizaron los años 1985, 1986, 1990, 1996, 2001 y 2007, registrando las variables especie, DAP $(\mathrm{cm})$, altura total (m), altura comienzo de copa (m), sanidad y forma, a todos los árboles mayores a $5 \mathrm{~cm}$ de DAP. Para la estimación de altura total se utilizó la función utilizada por Navarro (1997) [1]:

$$
\mathrm{HT}=9,252717+0,2523175 * \mathrm{DAP}
$$

donde $\mathrm{HT}=$ altura total del árbol $(\mathrm{m}), \mathrm{DAP}=$ diámetro a la altura del pecho $(\mathrm{cm})$.

Para efectos de obtener los productos que fueron definidos en base al mercado en Chile, se utilizó la función de ahusamiento polinomial [2] (Tapia 1988):

$\mathrm{D}_{\mathrm{i}} / \mathrm{DAP}=1,94627 *\left(\left(\mathrm{HT}-\mathrm{H}_{\mathrm{i}}\right) /(\mathrm{HT}-1,3)\right)-1,86688 *\left(\left(\mathrm{HT}-\mathrm{H}_{\mathrm{i}}\right)\right.$ $/(\mathrm{HT}-1,3))^{2}+0,904505 *\left(\mathrm{HT}-\mathrm{H}_{\mathrm{i}}\right) /(\mathrm{H}-1,3)^{3}$

donde $\mathrm{D}_{\mathrm{i}}=$ diámetro con corteza a la altura $\mathrm{H}_{\mathrm{i}}(\mathrm{cm})$, $\mathrm{DAP}=$ diámetro a la altura del pecho $(\mathrm{cm}), \mathrm{HT}=$ altura total del árbol (m).

Se determinó el índice de densidad relativa (IDR) de Curtis (1971), para lo cual se utilizó la función de tamaño máximo de densidad construida por Donoso et al. (2007).

Los productos en trozos se clasificaron de acuerdo a las dimensiones y calidades que demanda la industria en Chile, definiéndose como producto debobinable (P1) las trozas con diámetro mínimo de $35 \mathrm{~cm}$ y largo mínimo de $2,5 \mathrm{~m}$. Se consideraron secciones del fuste que no presentaban daños ni defectos de cancros, pudriciones, daños mecánicos, de insectos y abultamientos y que tenían forma circular, recta o inclinada, con muy ligeras desviaciones. No se admitieron muñones de un diámetro superior a $10 \mathrm{~cm}$, rajaduras ni ramas con pudrición sobre el tronco. Como producto aserrable (P2) se definieron trozas con diámetro mínimo de $20 \mathrm{~cm}$ y largo de $2,5 \mathrm{~m}$. Se consideraron secciones del fuste con daños locales de insectos no barrenadores y defectos mecánicos, la forma pudo presentar una sección transversal elíptica, el fuste debía ser recto o inclinado, se admitió la presencia de ramas y muñones. El producto metro ruma o leña (P3) se definió como el volumen total del árbol descontándole los productos P1 y P2, e incluyó en forma íntegra a aquellos individuos que por dimensiones, daños y forma no clasificaron el fuste para P1 y P2.

Análisis de rentabilidad. El análisis de rentabilidad se realizó mediante los indicadores valor actual neto (VAN), valor potencial del suelo (VPS) y la tasa interna de retorno modificada (TIRm). Se consideró el periodo desde los 30 a los 52 años del bosque secundario.

a) Valor actual neto (VAN). El criterio del valor actual neto consiste en obtener la diferencia de los beneficios y los valores actualizados de los costos (ecuación 3). Dado que los primeros se anotan con signo positivo y los segundos con negativo, la decisión es buena si VAN $>0$ y mala si VAN $<0$. Brealey y Myers (2001) señalan que el criterio del valor actual neto es aceptado 
por los economistas, y es el método más apropiado para calcular el beneficio de cualquier proyecto. El indicador valor actual neto se expresa como [3]:

$$
V A N=\sum_{j=0}^{r} \frac{B_{j}-C_{j}}{(1+i)^{r}}
$$

donde $\mathrm{Bj}=$ beneficio en un año $\mathrm{j}$ expresado en US\$, $\mathrm{C} j=$ costo en un año $\mathrm{j}$ expresado en USD, $\mathrm{r}=$ edad de corta final expresada en años, $\mathrm{i}=$ tasa de descuento expresada en valor decimal.

b) Valor económico del suelo (VES). La dinámica de los bosques naturales genera incertidumbre en su estructura después de la corta final, por lo tanto, para este estudio la aplicación del valor económico del suelo se realizó para efectos de comparar los resultados con otros estudios que utilizan este indicador. El valor económico del suelo calcula la rentabilidad del proyecto para infinitas rotaciones del bosque. Este es un indicador que tiene gran utilidad para determinar el monto máximo de dinero que se está dispuesto a pagar por un terreno destinado a infinitas rotaciones. La definición de valor económico del suelo [4] corresponde al mismo concepto de valor económico de cualquier bien de capital, que en términos prácticos es el valor actual de todos los beneficios futuros netos generados por el suelo (Chacón 1995):

$$
V E S=\frac{\sum_{j=0}^{r}\left(B_{j}-C_{j}\right)(1+i)^{r-j}}{(1+i)^{r}-1}
$$

donde $\mathrm{Bj}=$ beneficio en un año $\mathrm{j}$ expresado en USD, $\mathrm{C} j=$ costo en un año $\mathrm{j}$ expresado en US\$, $r=$ rotación expresada en años, $\mathrm{i}=$ tasa de descuento expresada en valor decimal.

c) Tasa interna de retorno modificada (TIRm). La determinación de una tasa interna de retorno única sólo es posible cuando a un saldo, o serie de saldos negativos, sigue una serie ininterrumpida de saldos positivos. Cuando los flujos financieros evaluados son de carácter heterogéneo, como es el caso de los proyectos forestales, este indicador genera soluciones múltiples, que limitan su uso en la toma decisiones. Para efectos de dar solución a esta restricción, Kierulff (2008) señala que la tasa interna de retorno modificada es el indicador más adecuado para evaluar flujos de estas características. Conceptualmente, la tasa interna de retorno modificada convierte el flujo financiero heterogéneo original en uno de carácter homogéneo, actualizando todos los costos a una tasa de financiamiento, y capitaliza todos los ingresos a una tasa de reinversión (Biondi 2006). La tasa de financiamiento utilizada fue la correspondiente al costo aplicado por las entidades financieras para los proyectos de inversión forestal en Chile, que en este caso es del $6 \%$ anual.

En relación al cálculo de la tasa de descuento, esta se calculó mediante el método CAPM (capital asset pricing model o modelo de valoración de activos financieros) (Copeland et al. 2005) y se calculó como lo detalla la siguiente ecuación [5]:

$$
\mathrm{i}=\mathrm{rf}+\beta[\mathrm{E}(\mathrm{Rm})]-\mathrm{rf}
$$

donde $\mathrm{i}=$ tasa de descuento en valor decimal; $\mathrm{rf}=$ tasa libre de riesgo, $\beta=$ riesgo sistemático del sector forestal; $\mathrm{E}(\mathrm{Rm})=$ esperanza de retorno del mercado. La tasa resultante para la industria forestal en Chile fue del $10 \%$.

La tasa obtenida a partir del cálculo de la tasa interna de retorno modificada corresponde a la tasa que hace al valor actual neto igual a cero (Brealey et al. 2006, Hartman y Schafrick 2004). Si ésta se compara con las tasas de descuento utilizadas para sensibilizar el valor actual neto, el criterio de decisión es que si la tasa es igual o mayor que ésta, el proyecto debe aceptarse y si es menor, conviene rechazarse (Sapag 2003).

Para el análisis de rentabilidad se aplicaron tasas de descuento de $6 \%, 8 \%$ y $10 \%$, una edad de corta final de 52 años y costos de administración de US\$ $40 \mathrm{ha}^{-1}$ año $^{-1}$ bajo dos escenarios, sin costo de administración antes de las intervenciones (S1), que corresponde a un caso en que el propietario administra el rodal desde el momento que aplica el raleo, y con costo de administración desde el establecimiento del rodal (S2). Los precios y costos de los productos de la industria en Chile para esta especie se muestran en el cuadro 1. Estos costos consideran rodales que presentan accesibilidad y que se ubican a una distancia de transporte no mayor a $200 \mathrm{~km}$ para los productos P1 y $\mathrm{P} 2$ y de $100 \mathrm{~km}$ para P3.

Cuadro 1. Precios y costos de producción en trozos puestos en planta.

Prices and production costs of Drimys winteri logs in

Chile.

\begin{tabular}{lcc}
\hline Producto & $\begin{array}{c}\text { Precio puesto } \\
\text { planta en trozos } \\
\left(\mathrm{US} \$ \mathrm{~m}^{-3}\right)\end{array}$ & $\begin{array}{c}\text { Costo puesto } \\
\text { planta en trozos } \\
\left(\mathrm{US} \$ \mathrm{~m}^{-3}\right)\end{array}$ \\
\hline Debobinable (P1) & 70 & 38 \\
Aserrable (P2) & 58 & 36 \\
Leña o metro ruma (P3) & 30 & 20 \\
\hline
\end{tabular}

P1 y P2: distancia de transporte $200 \mathrm{~km}$; P3: distancia de transporte $100 \mathrm{~km}$.

Fuente: Consulta a empresas en Chile durante el año 2008. 
Modelo de optimización silvicultural. Se determinó el método silvicultural óptimo para maximizar la rentabilidad y la producción física de estos bosques mediante un modelo de programación dinámica no probabilístico [6], que se expresó de la siguiente manera (Paredes y Brodie 1987):

$$
\int_{N}\left(Y_{n}\right)=\sum_{n=1}^{N} r_{n}\left(T_{n}\right)
$$

$\mathrm{X}_{\mathrm{n}}-\mathrm{T}_{\mathrm{n}}+\mathrm{G}_{\mathrm{n}-1}\left(\mathrm{Y}_{\mathrm{n}}\right)=\mathrm{X}_{\mathrm{n}-1} \quad(\mathrm{n}=1, \ldots, \mathrm{N}-1)$

$\mathrm{X}_{\mathrm{n}}-\mathrm{T}_{\mathrm{n}}=\mathrm{Y}_{\mathrm{n}} \quad(\mathrm{n}=1, \ldots, \mathrm{N})$

$\mathrm{X}_{\mathrm{N}}-\mathrm{T}_{\mathrm{N}}=0$ (condición de corta de protección en fajas)

donde,

$\int\left(Y_{n}\right)$ = valor de la función objetivo de una secuencia $\mathrm{Y}_{\mathrm{N}}$;

$r_{\mathrm{n}}\left(T_{\mathrm{n}}\right)=$ retorno generado en la etapa $\mathrm{n}$ cuando una decisión $\mathrm{T}_{\mathrm{n}}$ ha sido tomada;

$Y_{\mathrm{n}} \quad=$ vector que describe el rodal en la etapa $\mathrm{n}$ después que una decisión $\mathrm{T}_{\mathrm{n}}$ ha sido tomada en un rodal descrito por $\mathrm{X}_{\mathrm{n}}$ en esa etapa;

$X_{\mathrm{n}} \quad=$ vector que describe el estado del rodal en la etapa $\mathrm{n}$ después de que éste creció desde su estado $\mathrm{Y}_{\mathrm{n}-1}$;

$T_{\mathrm{n}} \quad=$ vector de posibles decisiones de manejo al estado $\mathrm{n}$ y que transforman el rodal $\mathrm{X}_{\mathrm{n}}$ en $\mathrm{Y}_{\mathrm{n}}$ generando un retorno $r_{n}\left(X_{n}, T_{n}\right)$;

$G_{\mathrm{n}+1}\left(Y_{\mathrm{n}}\right)=$ crecimiento del rodal $\mathrm{Y}_{\mathrm{n}}$ al estado $\mathrm{n}$ al que sigue un estado $\mathrm{n}+1$.

El problema de programación dinámica planteado incorpora los elementos básicos, indicados por Hillier y Lieberman (1982), definiendo las etapas (n) como estados de desarrollo de los bosques secundarios, con una decisión de la política requerida en cada etapa. Es decir, en cada etapa se tiene una variable (o vector) de decisión (Tn) y un número de estados o niveles de raleo (Yn) asociados a ella, que son las diversas condiciones posibles en las que el sistema podría estar en esa etapa del problema. El efecto de una decisión en cada etapa es transformar el estado actual en un estado (Xn) asociado con la etapa siguiente. Ello indica que los problemas de programación dinámica pueden interpretarse en términos de redes, donde cada nodo corresponde a un estado. El valor asignado a cada rama que conecta dos nodos puede interpretarse como la contribución a la función objetivo, hecha al ir de un estado a otro, en este caso, volumen extraído dado el estado actual. Una política óptima para las etapas restantes es independiente de la política adoptada en las etapas previas, o sea, que el estado actual del sistema proporciona toda la información necesaria acerca de su estado previo, para determinar la política óptima para cada estado en la última etapa.

\section{RESULTADOS}

Antecedentes dasométricos y tecnología silvicultural. Los bosques secundarios estudiados antes del manejo presentaban en promedio una edad de 30 años, un área basal de $57,7 \mathrm{~m}^{2} \mathrm{ha}^{-1}$, una densidad de 6.174 árboles $\mathrm{ha}^{-1}$, un DMC de $11,7 \mathrm{~cm}$ y una densidad relativa de $67 \%$. Los niveles de extracción en número de árboles para los tratamientos alcanzaban cifras de 64 a $88 \%$ y en términos de volumen las cifras variaron de 158 a $190 \mathrm{~m}^{3} \mathrm{ha}^{-1}$. Las densidades residuales fueron en promedio de 2.494, 1.094 y 611 árboles ha ${ }^{-1}$, y en términos de densidad relativa de 37,21 y $12 \%$ para los tratamientos de raleo moderado, fuerte (E2) y fuerte (E3), respectivamente. El tratamiento de raleo moderado fue intervenido nuevamente el año 1996, 10 años después de la primera intervención, cuando alcanzó el rodal un índice de densidad relativa de $49 \%$, extrayendo $60 \mathrm{~m}^{3} \mathrm{ha}^{-1}$ y reduciendo el índice de densidad relativa a $35 \%$, siendo equivalente su densidad residual al tratamiento de raleo fuerte (E2).

Después de 21 años de aplicadas las intervenciones de raleo, se pudo observar que el tratamiento testigo alcanzó mayor volumen y área basal al momento de la cosecha con respecto del tratamiento fuerte (E3), el que poseía $60 \%$ menos; pero se obtuvieron productos de mayor calidad que en el caso del testigo, el cual generó más del 50\% del volumen para uso de energía en calefacción (cuadro 2). En los tratamientos moderado (E1) y fuerte (E2) el volumen bruto total fue superior que el testigo, ya que se aprovecha un volumen que se pierde por mortalidad natural a causa de la fuerte competencia que se produce en estos bosques y al aumento de dimensión en diámetro de los árboles residuales. Los tratamientos de raleo generaron productos debobinables, observando que a una mayor intensidad existe un mayor volumen, a diferencia del testigo, que no presentó este producto, explicado por las menores dimensiones y calidad de los árboles (cuadro 2). La baja proporción de productos debobinables después de 21 años de ejecutado el raleo tardío indica que recién se inicia el aumento de la producción económica del sitio a causa del esfuerzo silvicultural. La tasa de crecimiento en DMC aumentó en el período evaluado, determinándose cifras de 10,6 a $22,3 \mathrm{~cm}$ en el raleo moderado, de 11,4 a $24,2 \mathrm{~cm}$ en el raleo fuerte (E2), de 10,7 a $26,5 \mathrm{~cm}$ en el raleo fuerte (E3); mientras que en el tratamiento sin manejo la cifra aumentó de 11 a $17 \mathrm{~cm}$.

Análisis de rentabilidad. En el escenario (S1), el de un propietario que administra el bosque secundario desde el momento que es realizado el tratamiento silvicultural, es decir, no se asignan costos de administración en los primeros 30 años, los indicadores de rentabilidad indicaron que todos los tratamientos fueron rentables en todas las tasas de descuento analizadas, para la condición evaluada. A la edad de corta de 52 años el tratamiento con un mayor valor actual neto a una tasa de $6 \%$ fue el raleo a 
Cuadro 2. Antecedentes dasométricos medios según edad*.

Dasometric characteristic of the treatments.

\begin{tabular}{|c|c|c|c|c|c|c|c|c|c|c|c|}
\hline Tratamiento & $\begin{array}{l}\text { Edad rodal } \\
\quad \text { (años) }\end{array}$ & $\begin{array}{l}\text { Densidad } \\
\left(\mathrm{N} \mathrm{ha}^{-1}\right)\end{array}$ & IDR & $\begin{array}{c}\mathrm{G} \\
\left(\mathrm{m}^{2} \mathrm{ha}^{-1}\right)\end{array}$ & $\begin{array}{c}\text { VT } \\
\left(\mathrm{m}^{3} \mathrm{ha}^{-1}\right)\end{array}$ & $\begin{array}{c}\mathrm{P} 1 \\
\left(\mathrm{~m}^{3} \mathrm{ha}^{-1}\right)\end{array}$ & $\begin{array}{c}\mathrm{P} 2 \\
\left(\mathrm{~m}^{3} \mathrm{ha}^{-1}\right)\end{array}$ & $\begin{array}{c}\mathrm{P} 3 \\
\left(\mathrm{~m}^{3} \mathrm{ha}^{-1}\right)\end{array}$ & $\begin{array}{l}\mathrm{DMC} \\
(\mathrm{cm})\end{array}$ & $\begin{array}{c}\text { VE Raleo } \\
(\mathrm{P} 3) \\
\left(\mathrm{m}^{3} \mathrm{ha}^{-1}\right)\end{array}$ & $\begin{array}{c}\text { V Total } \\
2007 \\
\left(\mathrm{~m}^{3} \mathrm{ha}^{-1}\right)\end{array}$ \\
\hline \multirow{4}{*}{$\begin{array}{l}\text { Raleo moderado } \\
\text { E1 (2 a } 3 \mathrm{~m})\end{array}$} & 30 & 7.006 & 61 & 62,4 & 395,8 & - & - & - & 10,6 & 158 & \multirow{4}{*}{458,1} \\
\hline & 31 & 2.494 & 37 & 36,2 & 216,7 & 0 & 4,03 & 212 & 13,6 & - & \\
\hline & 35 & - & 44 & - & - & - & - & - & - & 60 & \\
\hline & 52 & 928 & 40 & 45,1 & 240,1 & 10 & 75 & 155,1 & 22,3 & - & \\
\hline \multirow{4}{*}{$\begin{array}{l}\text { Raleo fuerte } \\
\text { E2 (3 m) }\end{array}$} & 30 & 5.678 & 58 & 56,6 & 361,0 & - & - & - & 11,4 & 190 & \multirow{4}{*}{441,3} \\
\hline & 31 & 1.094 & 21 & 20,4 & 125,2 & 0 & 13,6 & 111,6 & 15,3 & - & \\
\hline & 35 & - & 25 & - & - & - & - & - & - & - & \\
\hline & 52 & 828 & 43 & 39,5 & 251,3 & 26 & 95 & 130,3 & 24,2 & - & \\
\hline \multirow{4}{*}{$\begin{array}{l}\text { Raleo fuerte } \\
\text { E3 (4 m) }\end{array}$} & 30 & 5.367 & 50 & 47,8 & 309,1 & - & - & - & 10,7 & 186 & \multirow{4}{*}{365,8} \\
\hline & 31 & 611 & 12 & 10,9 & 65,8 & - & 8,4 & 57,4 & 15,3 & - & \\
\hline & 35 & - & - & - & - & - & - & - & - & - & \\
\hline & 52 & 506 & 32 & 27,9 & 179,8 & 20 & 68 & 91,8 & 26,5 & - & \\
\hline \multirow{4}{*}{$\begin{array}{l}\text { Testigo } \\
\text { E4 (sin manejo) }\end{array}$} & 30 & 6.174 & 67 & 57,7 & 354,7 & - & - & - & 11,7 & - & \multirow{4}{*}{439,4} \\
\hline & 31 & 6.174 & 67 & 57,7 & 354,7 & - & 12,1 & 342,6 & 11,7 & - & \\
\hline & 35 & - & 78 & - & - & - & - & - & - & - & \\
\hline & 52 & 3.483 & 64 & 78,3 & 439,4 & - & 118 & 321,4 & 17,0 & - & \\
\hline
\end{tabular}

*IDR: índice de densidad relativa, G: área basal por hectárea $\left(\mathrm{m}^{2} \mathrm{ha}^{-1}\right)$, VT: volumen por hectárea $\left(\mathrm{m}^{3}\right.$ ha $\left.{ }^{-1}\right)$, P1: volumen debobinable por hectárea $\left(\mathrm{m}^{3} \mathrm{ha}^{-1}\right), \mathrm{P} 2$ : volumen aserrable por hectárea $\left(\mathrm{m}^{3} \mathrm{ha}^{-1}\right), \mathrm{P} 3$ : metro ruma o leña $\left(\mathrm{m}^{3}\right.$ ha $\left.{ }^{-1}\right)$, DMC: diámetro medio cuadrático $(\mathrm{cm})$, VE Raleo: volumen extraído en los raleos $\left(\mathrm{m}^{3} \mathrm{ha}^{-1}\right)$, V Total 2007: volumen extraído en los raleos más el volumen total del año 2007 ( $\mathrm{m}^{3}$ ha $\left.{ }^{-1}\right)$.

$3 \mathrm{~m}$ con US\$ $2.724 \mathrm{ha}^{-1}$, un valor potencial del suelo de US\$ $3.771 \mathrm{ha}^{-1} \mathrm{y}$ una tasa interna de retorno modificada de $19 \%$ (cuadro 3 ).

Al comparar las cifras de rentabilidad entre tratamientos de raleo las diferencias no fueron importantes, lo que implica seleccionar o recomendar el tratamiento de raleo moderado, que reduce el riesgo de caída por viento y disminuye el costo de inversión inicial, generando un valor actual neto de US\$2.546, un valor potencial del suelo de US\$ 3.524 y una tasa interna de retorno modificada de $20 \%$ para una tasa de descuento de $6 \%$.

Al comparar el testigo con los tratamientos de raleo a la tasa de un $6 \%$, el valor actual neto fue al menos el doble con intervenciones; y a una tasa de $12 \%$ esta cifra subió al menos seis veces. La tasa interna de retorno modificada para la condición sin raleo alcanzó cifras de 13 a $12 \%$ (cuadro 3 ).

En el segundo escenario (S2), en el cual el propietario asigna costos de administración desde el momento en que se establece el bosque secundario, los indicadores de rentabilidad mostraron que al realizar la corta final a los 52 años no existió ningún tratamiento que haya obtenido un valor actual neto y valor potencial del suelo positivo para las tasas de descuento analizadas. Los resultados de la tasa interna de retorno modificada variaron de 4 a $8 \%$, para los tratamientos testigos y raleo fuerte (E3), respectivamente, cifras bastante inferiores al testigo del S1, que presentó una tasa interna de retorno modificada de 12 a $14 \%$. Los mejores tratamientos en este escenario correspondieron a los raleos fuerte (E2 y E3) con un $8 \%$ de tasa interna de retorno modificada (cuadro 3 ).

El modelo de programación dinámica determinó que el camino que optimiza el volumen total es no intervenir entre los 30 a 35 años y realizar un raleo moderado (E1) a los 35 años para aprovechar el volumen que se pierde por la alta mortalidad por competencia, generando un rendimiento en volumen a la edad de corta final de 52 años de $506 \mathrm{~m}^{3} \mathrm{ha}^{-1}$, un crecimiento medio anual en volumen de $9,7 \mathrm{~m}^{3} \mathrm{ha}^{-1} \mathrm{año}^{-1}$ y un valor actual neto de US\$ 1.318 $\mathrm{ha}^{-1}$ para una tasa de $6 \%$ para el escenario S1.

La maximización del valor actual neto se produjo con la senda óptima de un raleo fuerte (E2) a la edad de 30 años, dejando una densidad de 1.100 árboles ha ${ }^{-1}$ y realizando a la edad de 52 años la corta de protección en fajas, obteniendo un volumen de $441 \mathrm{~m}^{3} \mathrm{ha}^{-1}$, un crecimiento medio anual en volumen de $8,5 \mathrm{~m}^{3} \mathrm{ha}^{-1} \mathrm{año}^{-1} \mathrm{y}$ un valor 
Cuadro 3. Indicadores de rentabilidad por tratamiento y tasa de descuento.

Indicators of profitability per treatment and discount rate.

\begin{tabular}{|c|c|c|c|c|c|c|}
\hline Supuestos & Indicadores & $\mathrm{TD}(\%)^{*}$ & $\begin{array}{l}\text { Testigo } \\
\text { (E4) }\end{array}$ & Raleo fuerte (E3) & Raleo fuerte (E2) & $\begin{array}{l}\text { Raleo moderado } \\
\text { (E1) }\end{array}$ \\
\hline \multirow{9}{*}{ Supuesto 1 (S1) } & \multirow{3}{*}{$\begin{array}{l}\text { VAN } \\
(\text { US\$ ha-1) }\end{array}$} & 6 & 1.122 & 1.966 & 2.724 & 2.546 \\
\hline & & 8 & 641 & 1.751 & 2.344 & 2.177 \\
\hline & & 10 & 337 & 1.819 & 2.105 & 1.929 \\
\hline & \multirow{3}{*}{$\begin{array}{l}\text { VPS } \\
(\text { US\$ ha-1) }\end{array}$} & 6 & 1.553 & 2.721 & 3.771 & 3.524 \\
\hline & & 8 & 786 & 2.146 & 2.873 & 2.667 \\
\hline & & 10 & 384 & 2.074 & 2.400 & 1.929 \\
\hline & \multirow{3}{*}{$\operatorname{TIRm}(\%)$} & 6 & 12 & 18 & 19 & 20 \\
\hline & & 8 & 13 & 19 & 19 & 20 \\
\hline & & 10 & 13 & 20 & 20 & 21 \\
\hline \multirow{9}{*}{ Supuesto 2 (S2) } & \multirow{3}{*}{$\begin{array}{l}\text { VAN } \\
(\text { US\$ ha-1) }\end{array}$} & 6 & -2.001 & -996 & -398 & -671 \\
\hline & & 8 & -3.850 & -2.557 & -2.147 & -2.399 \\
\hline & & 10 & -6.203 & -4.721 & -4.435 & -4.682 \\
\hline & \multirow{3}{*}{$\begin{array}{l}\text { VPS } \\
\left(\mathrm{US} \$ \mathrm{ha}^{-1}\right)\end{array}$} & 6 & -2.002 & -1.379 & -551 & -929 \\
\hline & & 8 & -3.851 & -3.133 & -2.631 & -2.939 \\
\hline & & 10 & -6.204 & -5.382 & -5.056 & -5.338 \\
\hline & \multirow{3}{*}{ TIRm (\%) } & 6 & 4 & 7 & 7 & 6,62 \\
\hline & & 8 & 5 & 7 & 7 & 7,11 \\
\hline & & 10 & 5 & 8 & 8 & 7,51 \\
\hline
\end{tabular}

*TD: tasa de descuento, VAN: valor actual neto, VPS: valor potencial del suelo, TIRm: tasa interna de retorno modificada.

actual neto de US\$ 2.724 ha $^{-1}$ para una tasa de descuento del $6 \%$ en el escenario S1 analizado.

\section{DISCUSIÓN}

El manejo de los bosques cuyo objetivo es la producción de madera de calidad requiere intervenciones tempranas oportunas, que para el caso estudiado es cercana a los 12 años (Navarro et al. 1997). Además para ello es recomendable realizar raleos moderados periódicamente, lo que garantiza la estabilidad del rodal, más aún dadas las altas densidades y problemas de anclaje de estos bosques (Reyes et al. 2009). Sin embargo, éstos se encuentran en una etapa no oportuna para una primera intervención, lo que sugiere evaluar alternativas silvícolas factibles de implementar, aun cuando no sea ésta la opción ideal deseable.

Estos bosques son prácticamente monoespecíficos y los tratamientos privilegiaron como especie meta canelo, ello bajo el supuesto de que las condiciones postraleo facilitarían el establecimiento de un segundo dosel de tolerantes acompañando a $D$. winteri. Esto permitiría mayor complementariedad en el uso de los recursos y por ello una mayor productividad como lo plantea Kelty (1992), ya que las especies acompañantes de $D$. winteri tienen características de tolerancia, fenológicas y morfológicas distintas. Sin embargo, en condiciones postraleo para este sitio canelo prácticamente no regenera, lo que produce el desafío de establecerlo artificialmente o, en la corta final, ensayar manejo del sustrato, si se observa un importante número de individuos de buena calidad de especies de interés maderable como Laureliopsis philippiana y Amomyrtus luma, y en menor medida Saxegothaea conspicua, Weinmannia trichosperma y Eucryphia cordifolia (Navarro et al. 1999). Esto sugiere realizar estudios sobre el efecto de las intervenciones en la composición de especies o qué tipo de intervenciones realizar para priorizar canelo y otras especies de interés comercial.

El bosque secundario de $D$. winteri experimenta una muy buena respuesta al raleo en términos de crecimiento en todos los tratamientos realizados, a pesar de que tenía 30 años al momento de la intervención. Los tratamientos de raleo aumentan el tamaño medio del rodal en términos de diámetro, lo que se evidencia en la mayor oferta de productos debobinables a la edad de corta de 52 años, que 
para el caso del tratamiento testigo prácticamente no se logra. Esta situación ilustra que bosques de este tipo ubicados en zonas de mayor productividad, como es el caso de estudio, deben ser intervenidos mediante técnicas silviculturales para la producción de madera de alta calidad, de modo de generar un aumento de rentabilidad de estos bosques y su aprovechamiento integral. Al respecto, las evidencias de este estudio indican que un primer raleo moderado (E1) a la edad de 30 años dejando densidades de 2.500 y luego de 10 años un segundo raleo, con una densidad residual de 800 árboles por hectárea o densidades relativas de $35 \%$, permiten generar una rentabilidad similar a los tratamientos de mayor intensidad. Esto es recomendable ya que raleos sucesivos de menor intensidad reducen el riesgo de caída por viento y le dan mayor estabilidad al rodal y no producen una subutilización del sitio. Al respecto, el reciente estudio de Reyes et al. (2009) aporta mayores antecedentes de respuesta de raleos en bosques secundarios de $D$. winteri, no incorporando en su evaluación la oferta potencial de productos maderables.

La baja proporción de productos debobinables, después de 21 años de ejecutado el raleo, indica el inicio del aumento de la producción económica del sitio a causa del esfuerzo silvicultural, lo que sugiere esperar para realizar la corta final. Al respecto, Navarro et al. (1999) indican que a nivel predial en faenas forestales y raleos experimentados se ha encontrado una aceptable calidad interna para diámetros de hasta $60 \mathrm{~cm}$ en sitios de productividad media $\left(1,1\right.$ y $16,6 \mathrm{~m}^{3} \mathrm{ha}^{-1}$ por año, con índices de sitio asociados que varían entre 6,3 y $23,5 \mathrm{~m}$ de altura a una edad clave de 35 años), lo que sugiere para los renovales estudiados edades de rotación de al menos 80 años, dados los crecimientos diametrales obtenidos por Reyes et al. (2009).

La rentabilidad del manejo de estos bosques es positiva en el escenario en que el propietario no asume costos de administración antes de la intervención en todos los tratamientos analizados, que Souter (2000) lo explica por no existir costos de formación, pues estos ya están formados al momento en que se intervienen. En el escenario (2), sin embargo, los costos de administración incorporados generan rentabilidades negativas para los supuestos planteados.

El tratamiento que presenta mejores respuestas al raleo en términos económicos es el raleo fuerte (E2) con un valor actual neto de US\$ $2.724 \mathrm{ha}^{-1}$ a una tasa de $6 \%$ a la edad de corta de 52 años. Sin embargo, es recomendable operacionalmente reducir gradualmente la densidad para alcanzar el número de árboles meta para el período analizado (30 a 52 años), realizando al menos dos intervenciones antes de la corta final (raleo moderado), tecnología que genera un VAN $10 \%$ inferior que el raleo fuerte (E2). Al respecto, Navarro et al. (1999) en términos de ocupación de sitio indican que una primera intervención en estos bosques correspondiente al raleo mínimo implica dejar espaciamientos promedios de 2,5 m entre árboles, densidad que ocupa completamente la capacidad de carga del sitio, además señala que los fuertes vientos de la zona impiden recomendar operacionalmente tratamientos con mayores intensidades de raleo.

En estudios realizados en Eucalyptus globulus Labill (Guerra et al. 2007) se determinaron cifras inferiores a las obtenidas en esta investigación, lo cual indica el real potencial de estos bosques. Ello se explica que en el caso de este bosque secundario se considera un período de evaluación de 22 años, de los 30 a los 52 años de edad, y en el año cero no se consideran costos de formación del bosque y sí ingresos por raleo, lo que produce indicadores de rentabilidad superiores. Sin embargo, un trabajo de mayor rigurosidad debe incorporar estudios de rendimiento de productos en la industria para validar de esta forma la oferta potencial estimada en este estudio, además de precisar las condiciones de sitio y de homogeneidad de bosques a los cuales es factible aplicar la tecnología silvicultural planteada.

En bosques subtropicales de Uruguay con intervenciones sucesivas a partir de los 20 años y hasta la cosecha a los 80 años, Cubagge et al. (2007) determinaron un valor actual neto de -113 US\$ $\mathrm{ha}^{-1}$, un valor potencial del suelo de $-138 \mathrm{US} \mathrm{ha}^{-1}$ y una tasa interna de retorno de 3,6\%.

Hernández et al. (1996) determinan para bosques secundarios de $D$. winteri correspondientes a un sitio II según Calquín (1986) y orientados a la producción de madera de alto valor, cifras de 1.972 US\$ ha ${ }^{-1}$ para una tasa de

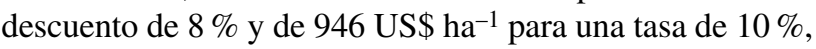
con una tasa interna de retorno aproximada de $15 \%$. La tecnología silvicultural propuesta comprende tres raleos, el primero a los seis años, el segundo a los 12, el tercero a los 36 años y una edad de cosecha de 45 años. Estas cifras son similares a las obtenidas en este estudio, a pesar de considerar tecnologías silviculturales distintas y edad de cortas diferentes, lo que demuestra la buena respuesta a intervenciones de este tipo de bosque y la necesidad de aplicar intervenciones tempranas y oportunas, lo que reduciría el tiempo de cosecha en 30 años.

Los niveles de densidad relativa alcanzados después de 21 años, con cifras mayores o iguales a $40 \%$, indican que es necesario realizar más intervenciones de raleo para alcanzar una plena ocupación del sitio y evitar que el rodal ocupe una zona de mortalidad por competencia, como ocurre en los raleos E1 y E2. Por su parte, el raleo fuerte (E3) alcanzó un índice de densidad relativa de $32 \%$ que indica que no es necesaria una nueva intervención, no obstante se debe velar por la estabilidad del rodal en los raleos como lo plantean Stathers et al. (1994). Al respecto, diversos autores recomiendan mantener los rodales en un nivel mínimo de $30 \%$ y máximo de $45 \%$ para regímenes de raleo para la producción de trozas para la industria de chapas y del aserrío (Drew y Flewelling 1979, Newton 1997, Saunders y Puettmann 2000, Shaw y Long 2007, Gezan et al. 2007).

Al comparar las sendas de optimización de valor actual neto y máximo volumen, se observa que el raleo es 
necesario en ambas situaciones, es decir, para maximizar el valor actual neto y el volumen total. La senda de máximo valor actual neto corresponde al raleo E2, el que reduce el índice de densidad relativa a $21 \%$, recuperándose a un $43 \%$ en el periodo de 21 años, esto es, se ubica en una zona de plena ocupación de sitio. El raleo fuerte (E3) después de la intervención deja al rodal en una zona de crecimiento libre, con un índice de densidad relativa de $12 \%$, no logrando en el periodo ocupar plenamente el sitio ya que solo alcanza un índice de densidad relativa de $32 \%$ a los 52 años; la ausencia de competencia permite que los árboles se desarrollen en crecimiento libre, produciendo mayores tamaños en menor tiempo, y generando de esta forma mayores precios. Sin embargo, la senda de máximo valor actual neto no ubica el rodal en el primer raleo en una zona de crecimiento libre o bajo un índice de densidad relativa de $15 \%$, ya que esto produce una subutilización del sitio, como lo plantean diversos autores (Drew y Flewelling 1979, Newton 1997, Saunders y Puettmann 2000).

Es importante tener presente el riesgo de caída de árboles por viento al aplicar raleos tardíos de alta intensidad. Al realizar un raleo por lo bajo intenso, como los tratamientos aplicados, en los cuales se dejan individuos dominantes y codominantes, y se eliminan las especies acompañantes del estrato inferior, se aumenta la vulnerabilidad de los árboles con respecto al viento, debido al gran tamaño de las copas remanentes, la alta relación altura/diámetro y la ausencia de un sostén lateral dado por los árboles vecinos (Stathers et al. 1994).

El análisis que se realiza no considera el perfil del propietario; sin embargo, la gradualidad de la reducción de las cifras de densidad es variable en el tiempo y depende, entre otros factores, de los ya indicados, de los objetivos del propietario y su capacidad para movilizar recursos.

\section{CONCLUSIONES}

El estudio evidencia que $D$. winteri es una especie que responde positivamente a raleos tardíos, aumentando su rentabilidad respecto de no intervenirlos. La intensidad de raleo fuerte (E2) es el que presenta mayor rentabilidad, mientras que el tratamiento de intensidad moderada presenta un mayor rendimiento en volumen total y una rentabilidad levemente inferior a los raleos de mayor intensidad.

El alto nivel de competencia de estos bosques expresado por su mortalidad natural requiere de intervenciones de raleo, ya sea para la maximización del volumen o del valor actual neto. Bajo este escenario el tratamiento recomendable de llevar a escala operacional es el raleo moderado, que obtiene rentabilidades levemente inferiores a los tratamientos de mayor intensidad, y que genera una menor mortalidad por efectos del viento.

La evaluación financiera del manejo en bosques secundarios debe considerar que éste se forma naturalmente, por lo que no existen gastos de administración antes de la primera intervención, no existe inversión inicial para que el bosque se forme y se generan ingresos con el primer raleo, produciéndose en ese caso rentabilidades positivas $y$ un aumento en volumen de los productos de mayor precio. En caso contrario, al considerar los costos de administración desde la formación del bosque, los resultados de rentabilidad son negativos.

La tecnología silvicultural tardía, recomendable para la producción de trozas de alta calidad en bosques secundarios de $D$. winteri de productividad media, debe considerar, para rentabilizar el esfuerzo silvicultural, rotaciones mayores a 52 años, raleos moderados y mantener densidades relativas no menores a $30 \%$ y máximas de $45 \%$.

\section{AGRADECIMIENTOS}

Al convenio de la Corporación Nacional Forestal y la Universidad Austral de Chile (CONAF-UACh) "Ecología y Silvicultura de los Bosques Nativos", el cual financió el establecimiento y evaluaciones de este ensayo, a las ingenieras forestales de la Universidad Católica de Temuco María José Sanhueza Pervan y Claudia Constanza Pinilla Sandoval del Laboratorio de SIG, Teledetección y Bosques.

\section{REFERENCIAS}

Acuña E, F Drake. 2003. Análisis del riesgo en la gestión forestal e inversiones silviculturales: una revisión bibliográfica. Bosque 24 (1): 113-124.

Biondi Y. 2006. The double emergence of the modified internal rate of return: The neglected financial work of Duvillard (1755-1832) in a comparative perspective. The European Journal of the History of Economic Thought 13(3): 311-335.

Brealey R, Myers S. 2001. Principios de finanzas corporativas. Madrid, España. Editorial McGraw-Hill. 805 p.

Brealey R, S Myers, F Allen. 2006. Principles of corporate finance (8th ed.). Boston, USA. McGraw-Hill/Irwin. 442 p.

Calquín R. 1986. Índices y clases de sitio para canelo (Drimys winteri (JR et Forster)) en la X Región. Tesis Ingeniero Forestal. Santiago, Chile. Facultad de Ciencias Agrarias y Forestales, Universidad de Chile. 125 p.

Chacón I. 1995. Decisiones económico-financieras en el manejo forestal. Talca, Chile. Ciencia y Tecnología. Editorial de la Universidad de Talca. 245 p.

CONAF (Corporación Nacional Forestal, CL), CONAMA (Comisión Nacional del Medio Ambiente, CL), Universidad Austral de Chile, Pontificia Universidad Católica de Chile, Universidad Católica de Temuco. 1997. Catastro y evaluación de los recursos vegetacionales nativos de Chile. Informe nacional con variables ambientales. Santiago, Chile. 88 p.

Copeland T, J F Weston, K Shastri. 2005. Financial theory and corporate policy. USA. Addison Wesley Publishing Company. 1024 p.

Curtis R. 1971. Tree area power function and related and density measures for douglas-Fir. Forest Science 17: 146-159. 
Cubbage F, P MacDonagh, J Sawinski, R Rubilar, PJ Donoso, A Ferreira, V Hoeflich, VM Olmos, G Ferreira, G Balmelli, J Siry, MN Báez, J Alvarez. 2007. Timber investment returns for selected plantations and native forests in South America and the Southern United States. New Forest 33(3): 237-255

Di Castri F, ER Hajek. 1976. Bioclimatología de Chile. Santiago, Chile. Imprenta-Editorial de la Universidad Católica de Chile. 129 p.

Donoso C. 1981. Tipos forestales de los bosques nativos de Chile. Investigación y desarrollo forestal (CONAF/ PNUD/ FAO). Santiago, Chile. Publicación FAO, Documento de Trabajo $\mathrm{N}^{\circ} 38.83$ p.

Donoso C. 1999. Silvicultura de los bosques nativos de Chile. Santiago, Chile. Editorial Universitaria. 421 p.

Donoso PJ, RD Nyland. 2005. Seeding density according to structure, dominance and understory cover in old-growth forest stands of the evergreen forest type in the coastal range of Chile. Revista Chilena de Historia Natural 78(1): 51-63.

Donoso P, D Soto, R Bertín. 2007. Size-density relationships in Drimys winteri secondary forests of the Chiloe Island, Chile: Effects of physiography and species composition. Forest Ecology and Management 1-3: 120-127.

Drew J, J Flewelling. 1979. Stand density management: an alternative approach and its application to Douglas-fir plantations. Forest Science 25(3): 518-532.

Emanuelli P. 1996. Bosque nativo, antecedentes estadísticos 19851994. Santiago, Chile. Corporación Nacional Forestal. 22 p.

Gezan S, A Ortega, E Andenmatten. 2007. Diagramas de manejo de la densidad para renovales de roble, raulí y coigüe en Chile. Bosque 28(2): 97-105.

Guerra E, M Herrera, F Drake. 2007. Rentabilidad de la fertilización al establecimiento de plantaciones de Eucalyptus globulus. Agrociencia 41(7): 797-804.

Hilliers F, G Lieberman. 1982. Investigación de operaciones. México D.F, México. McGraw-Hill. 833 p.

Hartman C, C Schafrick. 2004. The relevant internal rate of return. The Engineering Economist 49(2): 139-158.

Hernández M, C Donoso, M Romero. 1996. Variación genecológica de dos poblaciones de Drimys winteri (Forst.). Bosque 17(2): 65-75

INFOR (Instituto Forestal de Chile, CL). 2005. Estudio de mercado para madera de canelo en Estados Unidos y Europa. Santiago, Chile. INFOR. 92 p. (Informe técnico No 167).

Kelty M. 1992. Comparative productivity of monocultures and mixed-species stand. In Kelty M, B Larson, C Oliver eds. The ecology and silviculture of mixed-species stands. Dordrecht, The Netherlands. Kluwer Academic Publishers. p. $125-141$.

Kierulff H. 2008. MIRR: A better measure. Business Horizons 51:321-329.

Morales E, J Gayoso, A Ellies, R Gayoso, A Iroumé, R Mc Donald. 1988. Reconocimiento, evaluación y reclasificación de los suelos del sur de Valdivia costa. Valdivia, Chile. Universidad Austral de Chile. 22 p.

Navarro C. 1993. Estudio de raleo renovales de canelo, sector Hueicolla, Cordillera de la Costa de Valdivia. Instalación y evaluación del ensayo. Tesis Ingeniero Forestal. Valdivia, Chile. Facultad de Ciencias Forestales, Universidad Austral de Chile. 121 p.

Navarro C, C Donoso, V Sandoval, C González. 1997. Evaluación de raleos de canelo (Drimys winteri (Forst.)) en la Cordillera de la Costa de Valdivia, Chile. Bosque 18(2): 51-67.

Navarro C, C Donoso, V Sandoval. 1999. Los renovales de canelo. In Donoso C, A Lara eds. Silvicultura de los bosques nativos de Chile. Santiago, Chile. Editorial Universitaria. p. 341-377.

Newton P. 1997. Stand density management diagrams: Review of their development and utility in stand-level management planning. Forest Ecology and Management 98: 251-265.

Nyland R. 2002. Silviculture. Concepts and Applications. New York, USA. McGraw-Hill Companies. 633 p.

Oyarzún CE, R Godoy, A Sepúlveda. 1998. Water and nutrient fluxes in a cool temperate rainforest at the Cordillera de la Costa in southern Chile. Hydrological Processes 12: 1067-1077.

Paredes G, JD Brodie. 1987. Efficient specification and solution of the even-aged rotation and thinning problem. Forest Science: $33: 14-29$.

Reyes R, P Donoso, C Donoso, C Navarro. 2009. Crecimiento de renovales de Drimys winteri después de 16 años de aplicados distintos tratamientos de raleo en las cordilleras de Los Andes y de la Costa en Chile. Bosque 30(3): 117126.

Sapag N. 2003. Preparación y evaluación de proyectos. $3^{\text {a }}$ Edición. México D. F., México. McGraw Hill. 404 p.

Saunders MR, KJ Puettmann. 2000. A preliminary white spruce density management diagram for the Lake States. St. Paul, Minnesota, Department of Forest Resources, College of Natural Resources and Minnesota Agricultural Experiment Station. University of Minnesota. $14 \mathrm{p}$.

Shaw J, J Long. 2007. A density management diagram for longleaf pine stands with application to red-cockaded woodpecker habitat. Southern Journal of Applied Forestry 31(1): $28-38$

Souter R. 2000. Bosque nativo chileno: Un recurso para el desarrollo. Santiago, Chile. CONAF-GTZ. 92 p. (Proyecto Conservación y Manejo Sustentable del Bosque Nativo, CONAF-GTZ)

Stathers RJ, TP Rollerson, S Mitchell. 1994. Windthrow handbook for British Columbia forests. Research Program Working Paper 9401. Victoria, B.C., Canada. Ministry of Forests Research Program. 31 p.

Tapia E. 1988. Determinación de modelos de ahusamiento para renovales de canelo (Drimys winteri Forst). Tesis ingeniero forestal. Facultad de Ciencias Agrarias y Forestales, Universidad de Chile. 68 p.

Toral M, A Fratti, L González. 2005. Crecimiento estacional y rentabilidad de plantaciones forestales comerciales de pino radiata en suelos de trumao según método de establecimiento. Bosque 26(1): 43-54.

Recibido: 22.12.09

Aceptado: 17.07.10 